\title{
Changes in TL1A levels and associated cytokines during pathogenesis of diabetic retinopathy
}

\author{
ZHU-HONG ZHANG ${ }^{1,3^{*}}$, QING-ZHONG CHEN ${ }^{1 *}$, FENG JIANG ${ }^{1}$, TODD A. TOWNSEND ${ }^{2}$, CHUN-JIE MAO $^{1}$, \\ CAI-YUN YOU ${ }^{1}$, WEN-HUI YANG ${ }^{1}$, ZHI-YONG SUN $^{1}$, JIN-GUO YU $^{1}$ and HUA YAN ${ }^{1}$ \\ ${ }^{1}$ Department of Ophthalmology, Tianjin Medical University General Hospital, Tianjin 300052, \\ P.R. China; ${ }^{2}$ Division of Genetic and Molecular Toxicology, US Food and Drug Administration, \\ National Center for Toxicological Research, Jefferson, AR 72079, USA
}

Received September 3, 2015; Accepted October 4, 2016

DOI: $10.3892 / \mathrm{mmr} .2016 .6048$

\begin{abstract}
Tumor necrosis factor (TNF) ligand related molecule 1A (TL1A), also termed TNF superfamily member 15 and vascular endothelial growth inhibitor is important for tumorigenicity and autoimmunity. However, the function of TL1A in diabetic retinopathy (DR) remains to be elucidated. The present study established a diabetes mellitus (DM) rat model to investigate TL1A, vascular endothelial growth factor (VEGF), tumor necrosis factor- $\alpha(\mathrm{TNF}-\alpha)$ and interleukin- $1 \beta$ (IL-1 $\beta$ ) expression levels in the retina, vitreous and serum of rats with DM at different stages (1 month group, 3 month group and 6 month group). The present study determined that TL1A expression levels in the retina and vitreous from the DM 1 month group were significantly lower compared with the control group. However, TL1A levels in the retina and vitreous were significantly increased in advanced stages of DM compared with the control group. Furthermore, the levels of VEGF in the retina and vitreous were significantly higher in the DM groups compared with the control group. The expression levels of TNF- $\alpha$ and IL-1 $\beta$ in the retina and vitreous were significantly higher in DM 3 month and 6 month groups compared with the control group. It is of note that the expression levels of TL1A were significantly lower in the DM 1 and 3 month groups compared with the control group; however, they were significantly increased in the DM 6 month
\end{abstract}

Correspondence to: Dr Hua Yan, Department of Ophthalmology, Tianjin Medical University General Hospital, 154 Anshan Road, Tianjin 300052, P.R. China

E-mail: phuayan2000@163.com

Present address: ${ }^{3}$ Division of Genetic and Molecular Toxicology, US Food and Drug Administration, National Center for Toxicological Research, Jefferson, AR 72079, USA

\section{${ }^{*}$ Contributed equally}

Key words: diabetic retinopathy, TNF ligand related molecule 1A, vascular endothelial growth factor, tumor necrosis factor $\alpha$, interleukin-1 $\beta$ group compared with the DM 3 month group. The expression levels of VEGF, TNF- $\alpha$ and IL-1 $\beta$ in blood serum have been observed to exhibit similar expression change dynamics as those of the retina and vitreous. Therefore, these findings suggest that TL1A may be a protective factor of DR, and may provide a rationale for the development of novel therapeutic strategies to treat DR.

\section{Introduction}

Diabetic retinopathy (DR), classified into nonproliferative diabetic retinopathy and proliferative diabetic retinopathy (PDR), is an important diabetic microvascular complication, which has been identified as the leading cause of blindness in the working-age population worldwide (1). The prevalence of diabetes mellitus (DM) is growing and the number of patients with DM has been predicted to increase to 380 million by 2025 , with $\sim 4$ million people suffering from DR (2). A number of risk factors have been reported, including hyperglycemia, hypertension, dyslipidemia, diabetes duration, ethnic origin, pregnancy, puberty and cataract surgery $(3,4)$. Various additional systemic factors, including alcohol consumption, nephropathy, hypothyroidism, obesity, inflammation and endothelial dysfunction, have been reported to be associated with the development and progression of DR (2). Chronic hyperglycemia may initiate a series of physiological and biochemical changes that may result in microvascular damage and ultimately DR. Numerous signaling pathways, including vascular endothelial growth factor (VEGF) activation, inflammation, oxidative stress, protein kinase $\mathrm{C}$ activation and upregulation of the renin-angiotensin system (5), have been identified to contribute the development of DR. The present study focused on the role of tumor necrosis factor ligand related molecule $1 \mathrm{~A}$ (TL1A) in DR, which is hypothesized to exert its DR effects via inflammation.

TL1A, also termed tumor necrosis factor superfamily member 15 and vascular endothelial growth inhibitor, was previously identified as an anti-angiogenic cytokine that belonged to the TNF superfamily (6). TL1A may have a number of functions, including stimulation of dendritic cell maturation, activation of T-cells, inhibition of endothelial cell proliferation and endothelial progenitor cell (EPC) differentiation. In 
addition, previous studies have reported that TL1A is involved in inflammatory bowel disease as a Th1-polarizing cytokine (7), and suppressed tumor growth, endothelial cell proliferation and angiogenesis $(8,9)$. Furthermore, reduced expression levels of TL1A have been associated with unfavorable outcome for breast cancer patients (10). It has previously been demonstrated that TL1A was an important negative regulator for endothelial cell proliferation, which may result in inhibition of vasculogenesis. TL1A may inhibit the proliferation of endothelial cells by two mechanisms, the induction of early $G_{1}$ phase arrest and programmed cell death in proliferating cells (11). In addition, TL1A has been reported to inhibit vasculogenesis by regulation of soluble isoforms of VEGF receptor 1 (VEGFR1) (12). DR is a microvascular DM-associated vision disease with severe negative effects on the quality of life of patients. During DR, angiogenesis induces formation of new blood vessels and leads to the breakdown of the retinal barrier, which may ultimately result in blindness. As TL1A has been identified to contribute to vasculogenesis, the present study hypothesized that TL1A level may be important for the progression of DR and potentially provide a target for the generation of novel therapeutic and prognostic tools.

VEGF is a key factor that has been investigated in the pathogenesis of DR. The use of anti-VEGF therapy in diabetic macular edema, retinal angiogenesis and PDR has been effective; however, anti-VEGF therapies have transient benefits, as the edema recurs within a few weeks, and repeated injections are required (13-15). The role of inflammation in DR has been investigated extensively and has been demonstrated to be an important response in the development and progression of the disease pathology. A previous clinical study identified that elevated serum concentrations of tumor necrosis factor- $\alpha$ (TNF- $\alpha$ ), interleukin-1 $\beta$ (IL-1 $\beta$ ) and VEGF were associated with the presence and severity of diabetic retinopathy (16). Various VEGF-dependent therapeutic agents have been used clinically for the treatment of DR patients, including Macugen, Lucentis, and bevacizumab. However, these anti-VEGF therapeutic agent regimens included multiple treatments due to the recurring edema in patients with DR, requiring the development of novel therapeutic strategies for DR treatment. Previous studies indicated that VEGF may inhibit the expression of TL1A in ovarian cancer (17), and that expression levels of TL1A are upregulated by TNF- $\alpha$ and interleukin-1 (IL-1) in human umbilical vein endothelial cells (HUVECs) (18). In addition, previous studies have reported that TNF- $\alpha$ may contribute to the pathogenesis of DR, as TNF- $\alpha$ and IL- $1 \beta$ induced leukostatis and vascular permeability by the activation of macroglia and increased endothelial adhesion $(19,20)$. Elevated TNF- $\alpha$ and IL-1 $\beta$ expression levels were identified in the retina of diabetic animals and serum concentrations of VEGF, TNF- $\alpha$ and IL-1 $\beta$ were associated with the severity of DR $(20,21)$.

The present study investigated the expression levels of TL1A, VEGF, TNF- $\alpha$ and IL-1 $\beta$ in serum, the vitreous and retina in different stages of DR in rats, and discussed the roles of TL1A, VEGF, TNF- $\alpha$ and IL- $1 \beta$ in the pathogenesis of DR.

\section{Materials and methods}

Chemicals. TL1A (cat. no. ab85566), TNF- $\alpha$ (cat. no. ab6671), IL-1 $\beta$ (cat. no. ab9722) and VEGF (cat. no. ab46154) antibodies were obtained from Abcam (Cambridge, MA, USA). Enzyme linked immunosorbent assay (ELISA) kits were obtained from R\&D Systems, Inc. (Minneapolis, MN, USA). The Immunocruz ${ }^{\circledR}$ staining system was obtained from Santa Cruz Biotechnology, Inc. (Dallas, TX, USA).

Animal model. A total of 80 male Wistar rats (weight, 200-250 g; age, 6 weeks) were obtained from Military Medical Academy of China (Beijing, China). The rats were individually housed in a temperature $\left(21-23^{\circ} \mathrm{C}\right)$ - and humidity (40-70\%)-controlled room with a 12-h light/dark cycle, and were provided with food and water. All the rats were handled according to the National Institutes of Health Guide for the Care and Use of Laboratory Animals (22) and the procedures were approved by the Animal Care and Use Committee of Tianjin Medical University (Tianjin, China). The rats were randomly assigned to four groups: i) Control group ( $\mathrm{n}=20$, no-treatment); ii) DM 1 month group $(\mathrm{n}=20)$; iii) DM 3 month group ( $n=20)$; and iv) DM 6 month group $(n=20)$. Diabetes was induced with an intraperitoneal streptozotocin injection ( $60 \mathrm{mg} / \mathrm{kg}$ body weight). Rats with blood sugar levels between 16.5 and $22.5 \mathrm{mM}$ after 3 days of the administration of streptozotocin were assigned to the diabetic group $(n=60)$ and included in the experiment. Blood glucose levels were quantified once a week with a blood glucose monitor (LifeScan, Inc., Milpitas, CA, USA) and small insulin (Bantus) doses (4 UI/kg) were administered when necessary, in order to maintain the blood glucose levels between 16.5 and $22.5 \mathrm{mM}$.

Immunohistochemistry. Formalin-fixed paraffin retina sections $(5 \mu \mathrm{m})$ were stained with hematoxylin and eosin (H\&E). Immunohistochemistry was performed as previously described (23). Briefly, the staining of TL1A, VEGF, TNF- $\alpha$ and IL-1 $\beta$ was performed on retina tissue by using a monoclonal antibody against TL1A, VEGF, TNF- $\alpha$ and IL- $1 \beta$ at a dilution of 1:100. The Immunocruz ${ }^{\circledR}$ staining system was used, following the manufacturer's protocol. Staining-positive cells were analyzed randomly in 6 fields at a magnification of $x 400$. Results were calculated by counting the positively stained cells. Images were captured using an Olympus BX51 fluorescent microscope (Olympus Corporation, Tokyo, Japan).

ELISA. Blood samples from each rat was collected from the femoral vein and stored in blood collection tubes without anticoagulants, and placed upright at room temperature for $20 \mathrm{~min}$, in order to allow the blood to clot naturally and the serum was separated by centrifugation at $1,000 \times \mathrm{g}$ at $20^{\circ} \mathrm{C}$ for $15 \mathrm{~min}$. Vitreous samples were collected using $1 \mathrm{ml}$ syringes. Serum and vitreous samples were maintained at $-80^{\circ} \mathrm{C}$ until biochemical assays were performed. Serum and vitreous TL1A, VEGF, TNF- $\alpha$ and IL-1 $\beta$ levels were quantified using ELISA kits according to the manufacturer's protocol. TL1A, VEGF, TNF- $\alpha$ and IL- $1 \beta$ levels were determined by reading the absorbance at $450 \mathrm{~nm}$ and the concentration of the cytokines in the samples was demonstrated by comparing the optical density of the samples to the standard curve.

Statistical analysis. SPSS version 17.0 (SPSS, Inc., Chicago, IL, USA) was used for all statistical analyses. Data are presented 
as the mean \pm standard deviation. Differences between groups were analyzed using one-way analysis of variance or Student's paired t-test. $\mathrm{P}<0.05$ was considered to indicate a statistically significant difference.

\section{Results}

$H \& E$ staining of retina DM model. To confirm that the DM model was successfully established, H\&E staining was performed and the normal physiology of cell layers were observed in the control (Fig. 1A) and DM 1 month groups (Fig. 1B). By contrast, the DM 3 month group exhibited signs of retinal edema, such as disorder in cell layers, decrease of ganglion cells and the presence of microvessels in the inner plexiform layer (IPL), as denoted by arrows (Fig. 1C). It is of note that the DM 6 month group exhibited severe retina edema, thinner retina, deep staining of ganglion cells and increased microvessels in the IPL (Fig. 1D). In addition, the inner nuclear layer (INL) was in structural disarray, edema was apparent, fat vacuoles were visible (indicated with arrowheads; Fig. 1D) and layers of the retina were difficult to distinguish. This demonstrated that the streptozotocin injection successfully induced the DR model in rats. Notably, changes to the retina due to the pathology of DR first observed in DM rats in the 3 month group. Typical pathology changes of DR were clearly visible in the retina of DM rats at 6 months.

Changes of TL1A levels in retina and vitreous of rats with different stages of DM. Previous studies have reported TL1A to be a negative regulator of proliferation for endothelial cells, resulting in inhibition of vasculogenesis $(7,11,12)$. As DR is a microvascular DM-associated vision disease involving vasculogenesis, the present study investigated the levels of TL1A in the retina of rats with DM by immunohistochemical analysis. It was demonstrated that TL1A was expressed in retina tissues of the control groups, including the INL and ganglion cell layer (Fig. 2A). TL1A levels in the DM 1 month group were significantly lower compared with the control group $(\mathrm{P}<0.05$; Fig. 2B); however, they were increased to near baseline levels in the DM 3 month group. In the DM 6 month group, the TL1A levels were significantly higher compared with the control group $(\mathrm{P}<0.05$; Fig. 2B). ELISA was used to confirm the changes of the TL1A expression levels in the vitreous of rats at different stages of DM. These assays exhibited the same changes of TL1A expression levels observed in the retina. Briefly, the levels of TL1A were significantly lower in the DM 1 month and 3 month groups compared with the control group ( $\mathrm{P}<0.05$; Fig. 2C). In addition, TL1A levels were significantly higher in the DM 6 month group compared with the control $(\mathrm{P}<0.05$; Fig. 2C). Thus, it is possible that the expression levels of TL1A may be regulated by other cytokines.

Levels of VEGF are upregulated in the retina and vitreous of rats with DM. It has previously been reported that VEGF may inhibit the expression of TL1A (17). Therefore, the present study conducted immunohistochemistry experiments in the retina and ELISA in the vitreous to determine the expression levels of VEGF at different stages of DM. Expression levels of VEGF in the DM groups were all significantly higher compared with the control group, and the levels increased with the progression of
$\mathrm{DR}$ in the retina and vitreous $(\mathrm{P}<0.05$; Fig. 3$)$. This result was consistent with our previous study (24), which demonstrated that the level of VEGF in patients with DR was significantly higher compared with healthy persons. However, in the present study, this trend did not negatively correlate with TL1A levels in the retina and vitreous, suggesting that the expression of TL1A may be regulated by additional factors.

Ratios of TL1A/VEGF in retina and vitreous of rats with $D M$. As TL1A and VEGF have opposing functions in microvascular endothelial cells, the ratio of TL1A/VEGF in the retina and vitreous are important to the development and progression of DR. Therefore, quantification of the immunohistochemistry experiments in retina and ELISA assays in vitreous were used to calculate the ratio of TL1A/VEGF. It was demonstrated that the ratio of TL1A/VEGF in the retina of DM 1 month rat group was significantly reduced to $35.37 \%$ compared with the control $(\mathrm{P}<0.05$; Fig. $4 \mathrm{~A})$. The ratio of retinal TL1A/VEGF was increased in the DM 3 month group and peaked in the DM 6 month group. However, this peak was significantly lower compared with the control group $(\mathrm{P}<0.05$; Fig. 4A). Consistent with the observations obtained from the retina, the ratio of TL1A/VEGF in the vitreous of rats with DM 1 month significantly decreased to $61.77 \%$ compared with the control group $(\mathrm{P}<0.05$; Fig. 4B). The ratio of vitreous TL1A/VEGF was significantly increased only in the DM 6 month group, which remained significantly lower than that of the control group $(\mathrm{P}<0.05$; Fig. 4B). These data demonstrated that although the ratio of TL1A/VEGF increased during the development and progression of DR, it was consistently lower in DM groups compared with the control group. Therefore, it is possible that additional factors are involved in the pathophysiology of DR.

Levels of TNF- $\alpha$ and $I L-1 \beta$ are upregulated in the retina and vitreous of rats with DM. Previous studies have reported that $\mathrm{DM}$ increased the release of retinal inflammatory mediators, including IL-1 $\beta$, TNF- $\alpha$, VEGF, intercellular adhesion molecule 1 (ICAM-1) and angiotensin II (16), and the expression of TL1A was upregulated by TNF- $\alpha$ and IL-1 $\beta$ (18). To investigate the association between the expression of TL1A and TNF- $\alpha$ and IL- $1 \beta$ levels, immunohistochemistry analysis of the retina and ELISA assays in vitreous were performed. The immunochemistry result indicated a time-dependent increase in TNF- $\alpha$ expression levels (Fig. 5A) and IL-1 $\beta$ (Fig. 5B) during the development and progression of DR. The ELISA assays were consistent with the immunohistochemistry as the expression levels of TNF- $\alpha$ and IL-1 $\beta$ were significantly higher in DM 3 month and 6 month groups compared with the control group ( $\mathrm{P}<0.05$; Fig. $5 \mathrm{C}$ and $\mathrm{D})$. These data suggested that the increased TNF- $\alpha$ and IL- $1 \beta$ levels may contribute to the increased expression of TL1A.

Levels of TL1A, VEGF, TNF- $\alpha$ and IL-1 $\beta$ in serum samples from rats with $D M$. Previous studies have reported elevated serum concentrations of IL- $1 \beta$, TNF- $\alpha$, and VEGF correlate with the severity of DR $(19,21)$. In order to investigate the association between levels of TL1A, VEGF, TNF- $\alpha$ and IL-1 $\beta$ and the pathogenesis of DR, their expression levels in serum from DM rats were quantified using ELISA assays. 
A

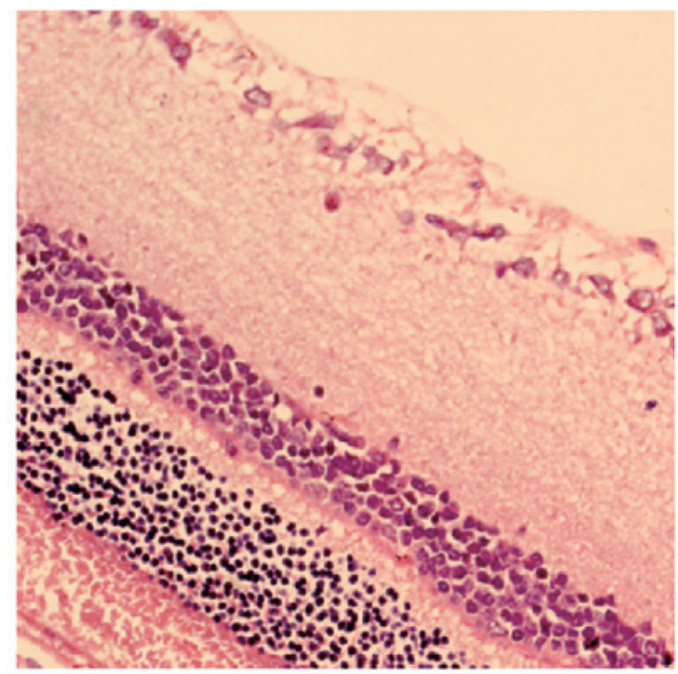

C

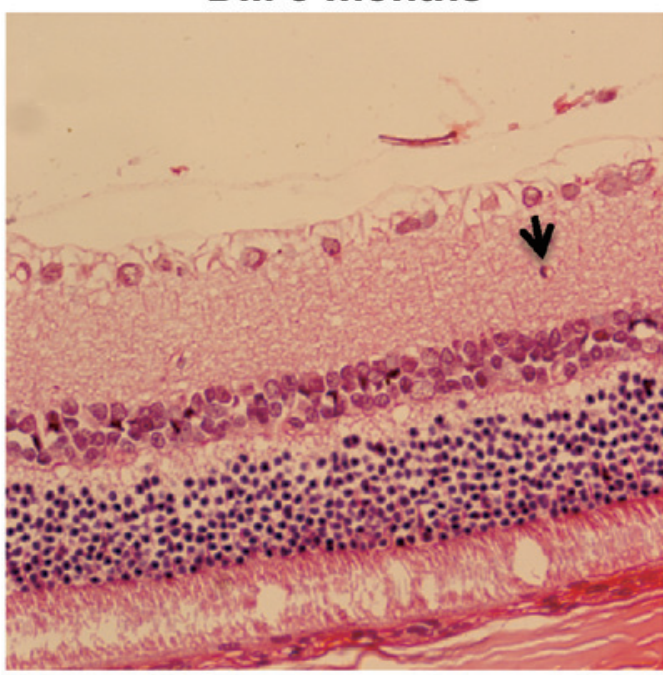

B

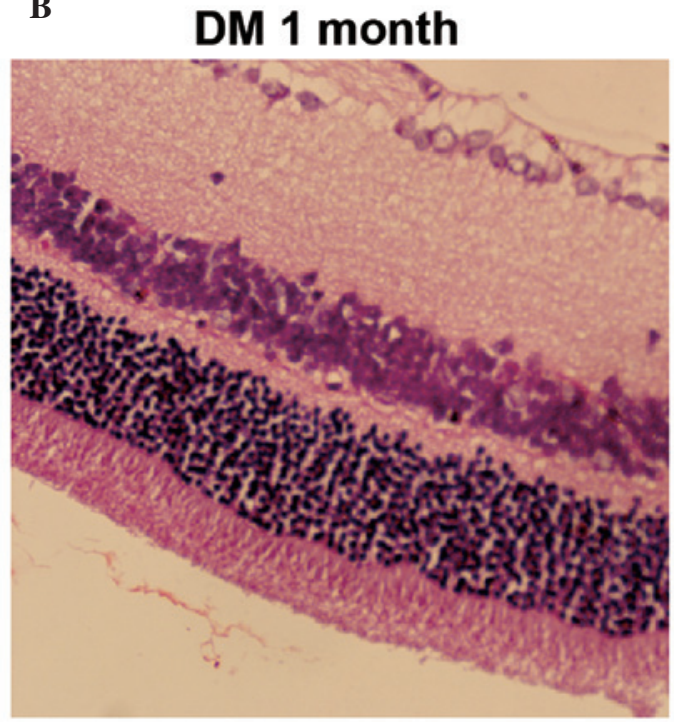

D

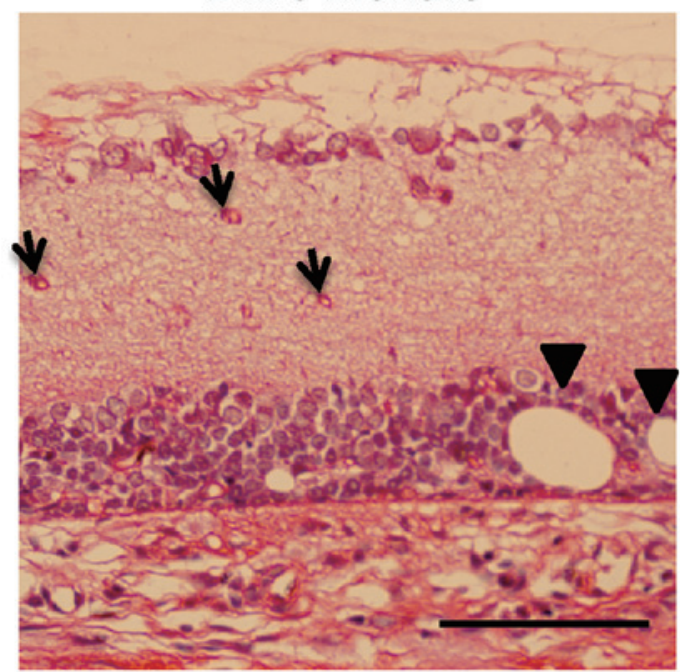

Figure 1. H\&E staining of the retina in the DM model. Retina H\&E examination in (A) control, (B) DM 1 month, (C) DM 3 month and (D) DM 6 month groups. Microvessels in the inner plexiform layer are denoted by arrows and fat vacuoles are denoted by arrrowheads. Scale bar, $100 \mu \mathrm{m}$. Ctrl, control; DM, diabetes mellitus; H\&E, hematoxylin \& eosin.

The changes of the TL1A, VEGF, TNF- $\alpha$ and IL-1 $\beta$ levels in serum indicated similar expression change dynamics as those observed in the retina and vitreous. Specifically, the expression levels of TL1A were significantly lower in DM 1 and 3 month groups compared with the control group $(\mathrm{P}<0.05$; Fig. 6). However, they significantly increased in DM 6 month group compared with the DM 3 month group (Fig. 6), which was different from the changes of TL1A levels in the retina and vitreous. The VEGF expression levels were significantly higher in all three DM groups compared with the control group $(\mathrm{P}<0.05$; Fig. 6$)$, which was consistent with VEGF changes observed in the retina and vitreous. The expression levels of TNF- $\alpha$ and IL-1 $\beta$ were significantly higher in DM 3 month and 6 month groups compared with the control group, which was consistent with changes observed in the retina and vitreous $(\mathrm{P}<0.05$; Fig. 6$)$. These results suggest that the levels of TL1A, VEGF, TNF- $\alpha$ and IL- $1 \beta$ in the serum may be associated with the changes observed in the retina and vitreous.

\section{Discussion}

Previous studies have reported that the pathogenesis of DR may be associated with microvascular occlusion and leakage, neuronal dysfunction and inflammation $(4,16)$. VEGF has been identified as a proinflammatory factor that affected endothelial tight junction proteins, resulting in the extravasation of fluid and retinal edema (25), and has been considered to be a key molecule for the pathogenesis of DR. TL1A has been identified as an important cytokine that may be involved in various inflammatory responses and hyperplasia of microvascular diseases $(9,26)$. The present study demonstrated that TL1A may act as a protective factor in the pathogenesis of DR. The retina histopathology (H\&E stain) results revealed that retina pathology changes that may be attributed to DR were evident in the rats from the DM 3 month group and typical pathology changes of DR were also observed in DM 6 month group. Subsequently, it was determined that the expression levels of TL1A were lower in the early stages of DM compared with the 
A

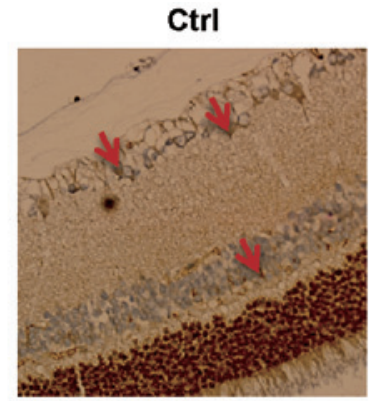

DM 1 month

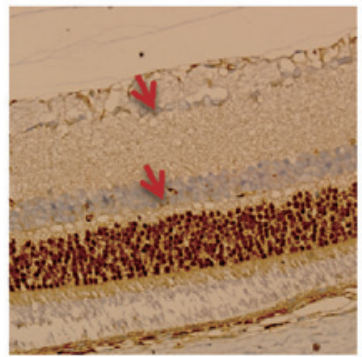

DM 3 month

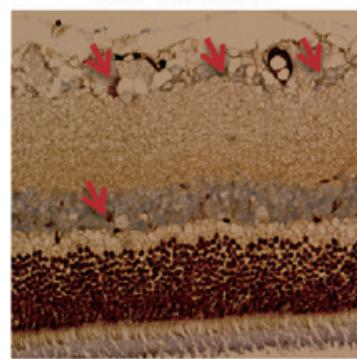

DM 6 month

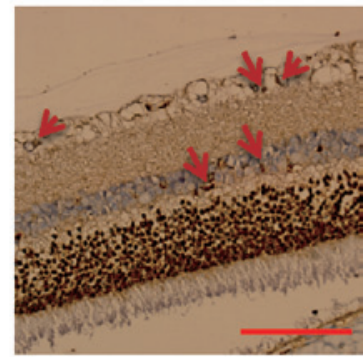

B

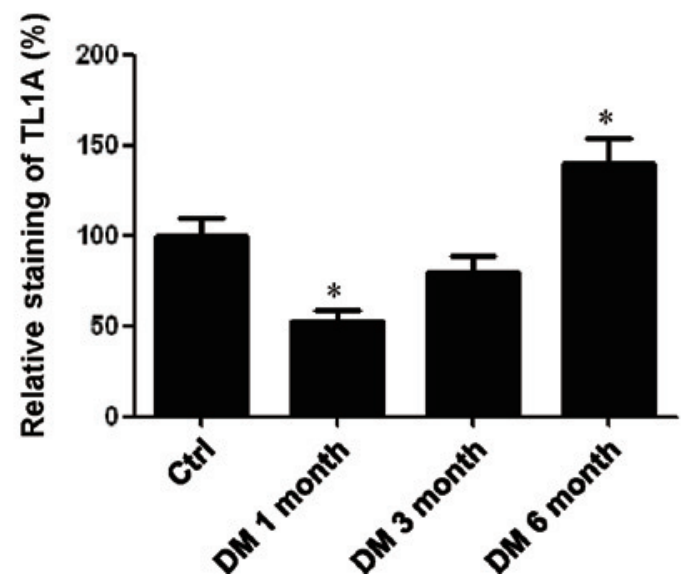

C

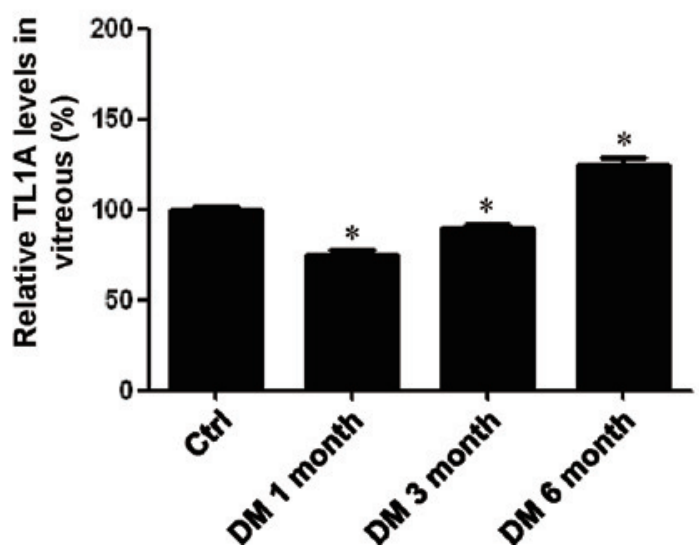

Figure 2. TL1A expression in retina and vitreous from DM rats. (A) Immunohistochemistry of control, DM 1 month, DM 3 month and DM 6 month groups. Arrows indicate TL1A expression. (B) Quantification of TL1A staining levels in the retina from the different rat treatment groups. (C) ELISA analysis of TL1A levels in vitreous from rats in the different treatment groups. Data are presented as the mean \pm standard deviation. ${ }^{*} \mathrm{P}<0.05$ vs. the control group. Scale bar, $100 \mu \mathrm{m}$. TL1A, tumor necrosis factor ligand related molecule 1A; Ctrl, control; DM, diabetes mellitus.

A

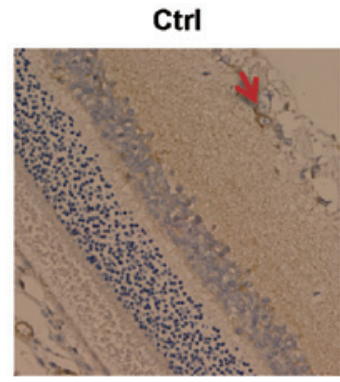

DM 1 month

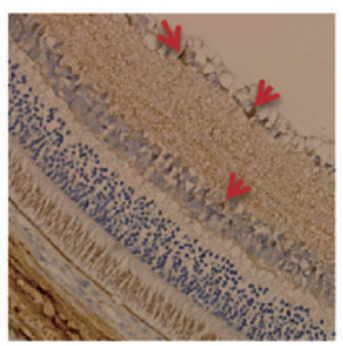

DM 3 month

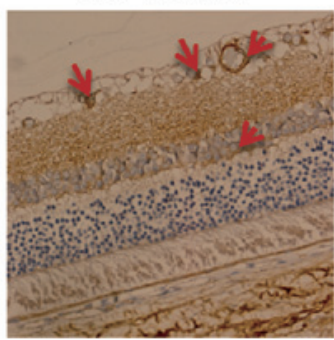

DM 6 month

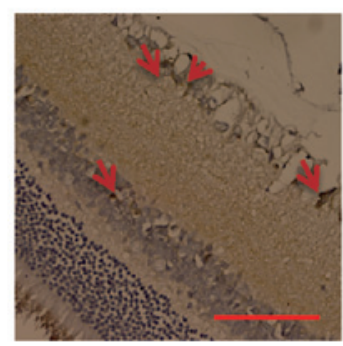

C

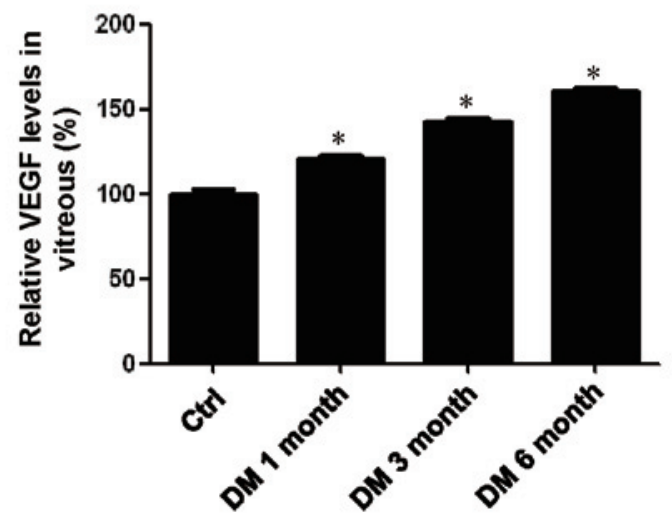

Figure 3. Levels of VEGF in retina and vitreous in DM rats. (A) Immunohistochemistry in control, DM 1 month, DM 3 month and DM 6 month groups. The arrows indicate areas of marked VEGF expression. (B) Quantification of VEGF staining levels in retina from rats. (C) ELISA analysis of VEGF levels in vitreous from rats in the different treatment groups. Data are presented as the mean \pm standard deviation. " $\mathrm{P}<0.05$ vs. control group. Scale bar, $100 \mu \mathrm{m}$. Ctrl, control; DM, diabetes mellitus; VEGF, vascular endothelial growth factor. 
A

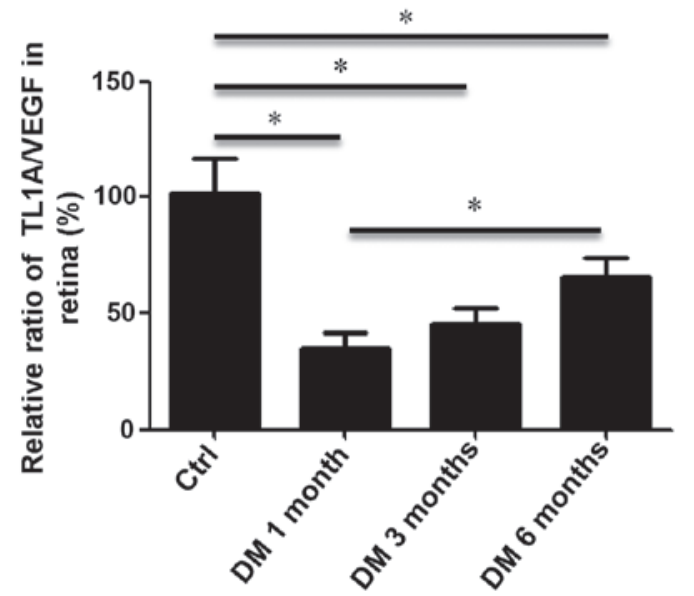

B

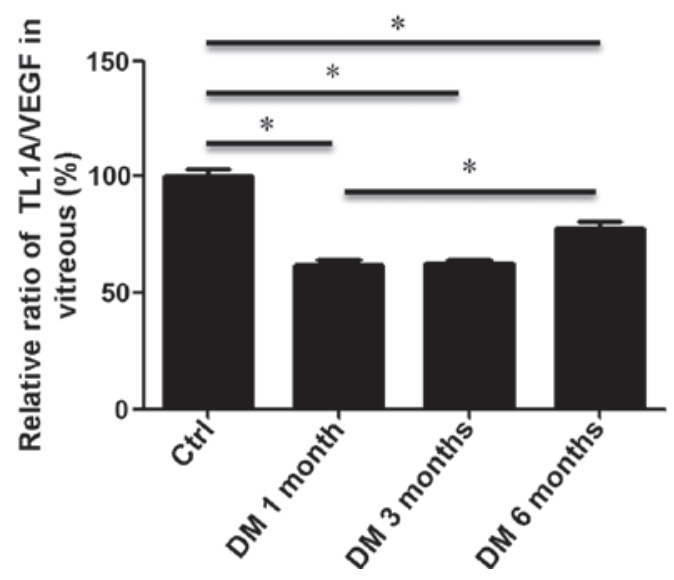

Figure 4. (A) Immunohistochemistry quantification of TL1A/VEGF ratio in rat retina. (B) ELISA quantification of TL1A/VEGF ratio in vitreous. Data are presented as the mean \pm standard deviation. ${ }^{*} \mathrm{P}<0.05$ vs. control group. TL1A, tumor necrosis factor ligand related molecule $1 \mathrm{~A}$; VEGF, vascular endothelial growth factor; Ctrl, control; DM, diabetes mellitus.

A

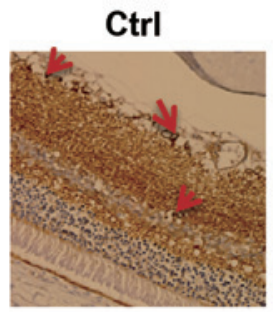

B

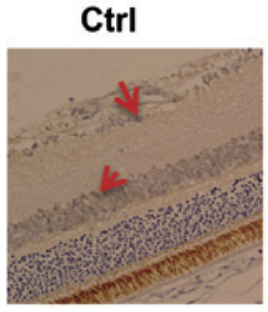

DM 1 month

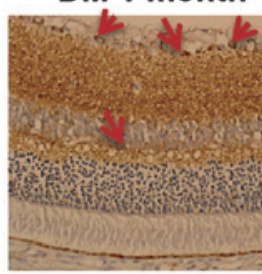

DM 1 month
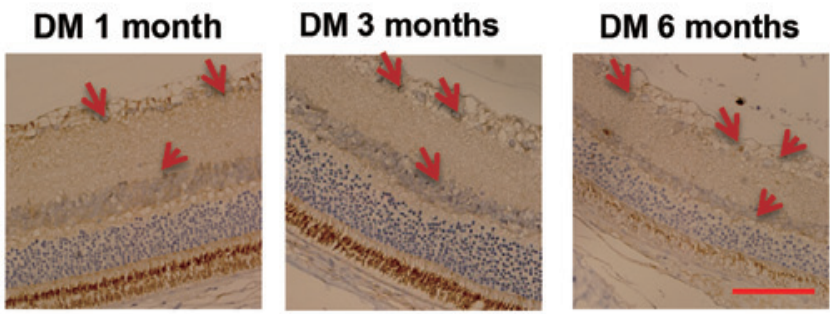
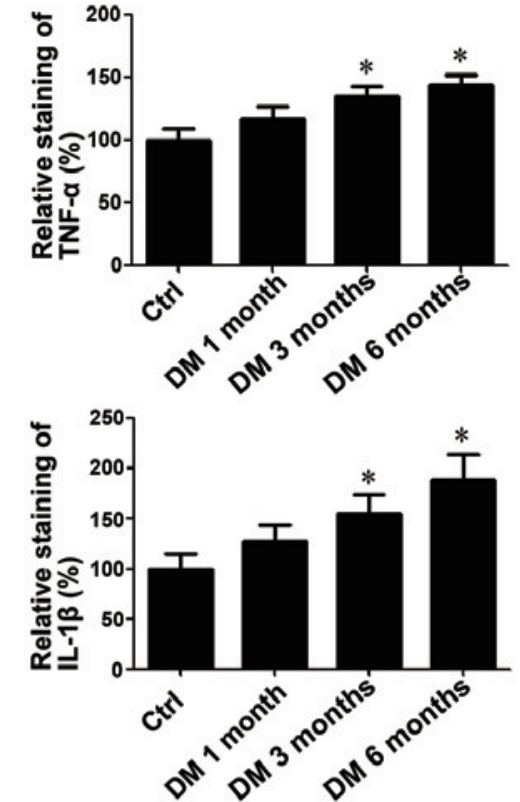

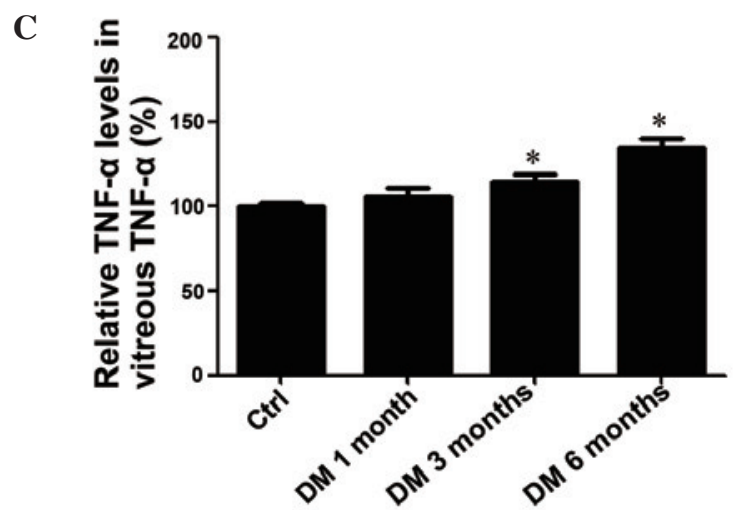

D

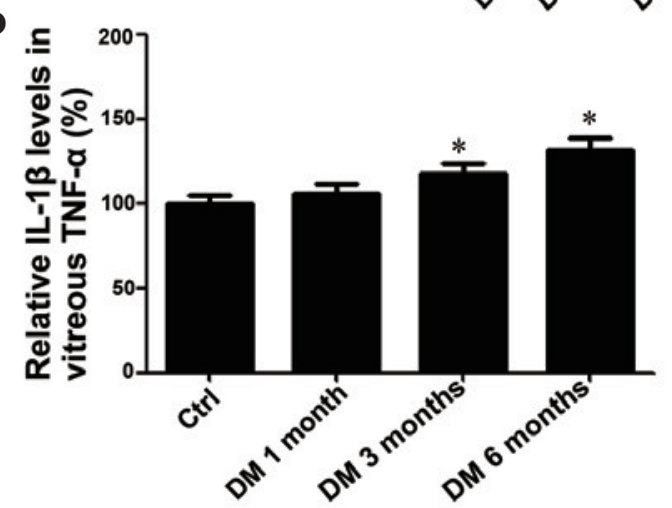

Figure 5. Levels of TNF- $\alpha$ and IL-1 $\beta$ in retina and vitreous in DM rats. Immunohistochemistry images and quantification of (A) TNF- $\alpha$ and (B) IL-1 $\beta$ levels in retina from rats in the different treatment groups. The arrows indicate TNF- $\alpha$ and IL-1 $\beta$ expression. Scale bar, $100 \mu \mathrm{m}$. ELISA analysis of (C) TNF- $\alpha$ and (D) IL-1 $\beta$ levels in vitreous from rats in the different treatment groups. Data are presented as the mean \pm standard deviation. "P $<0.05$ vs. control group. TNF- $\alpha$, tumor necrosis factor $\alpha$; IL-1 $\beta$, interleukin-1 $\beta$; Ctrl, control; DM, diabetes mellitus.

control group, and increased in groups with DR (DM 3 and 6 month groups). Consistent with disease progression, levels of VEGF were higher in the DM groups compared with the control group. Additionally, the levels of TNF- $\alpha$ and IL-1 $\beta$, 


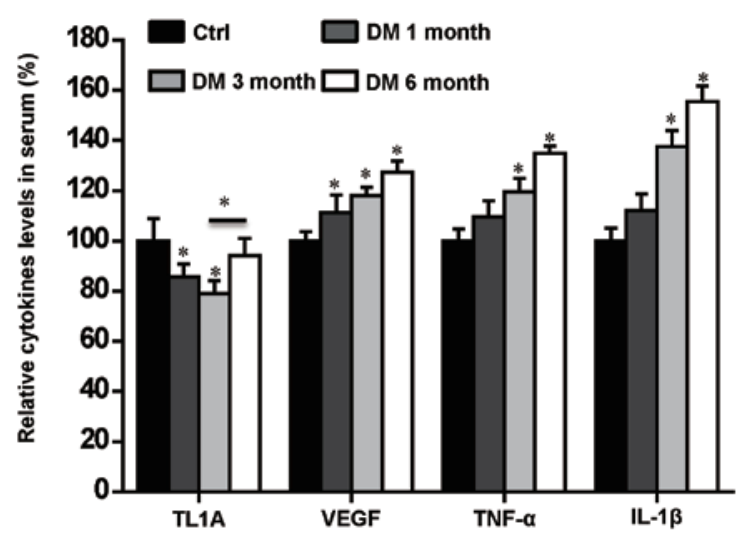

Figure 6. Levels of TL1A, VEGF, TNF- $\alpha$ and IL-1 $\beta$ in blood serum from DM rats was determined using ELISA analysis Data are presented as the mean \pm standard deviation. ${ }^{*} \mathrm{P}<0.05$ vs. control group, or as indicated TL1A, tumor necrosis factor ligand related molecule 1A; VEGF, vascular endothelial growth factor; TNF- $\alpha$, tumor necrosis factor $\alpha$; IL-1 $\beta$, interleukin-1 $\beta$; Ctrl, control; DM, diabetes mellitus.

which had been reported to upregulate the expression of TL1A were investigated in the DM groups. The present study determined that the expression levels of TNF- $\alpha$ and IL-1 $\beta$ were higher in the DM groups compared with the control group. Therefore, it is possible that the ratio of TL1A/VEGF may contribute to the development and progression of DR. Furthermore, the expression of TL1A may be regulated by various factors, including VEGF, TNF- $\alpha$ and IL-1 $\beta$.

TL1A, which belongs to TNF superfamily, has been expressed primarily in endothelial cells as a negative modulator of angiogenesis and may be induced in response to inflammatory stimuli $(9,26,27)$. Previous studies have reported that TL1A suppressed the ability of capillary formation in vitro, which was induced by fibroblast growth factor-2 (FGF2) or VEGF in adult bovine aortic endothelial cells (27). Additionally, TL1A inhibited $~ 50 \%$ of the neovascularization induced by either FGF2 or VEGF in vivo. A recent study Qi et al (12) determined that TL1A inhibited vasculogenesis by regulating the expression levels of the VEGFR1. They also reported that TL1A inhibited VEGF-driven, EPC-supported vasculogenesis in a murine matrigel implant model (12). Therefore, it is possible that TL1A may be important in the development and progression of DR, which may be characterized by inflammation and neovascularization driven by pro-angiogenesis growth factors and cytokines. The current study investigated the association between the expression of TL1A and the development and progression of DR, by quantifying the expression levels of TL1A in the retina and vitreous of DM rats. It was determined that TL1A levels were significantly lower in the early stages of DM and started to increase in the DM 3 month group, which was consistent with the features of the early stage of DR observed in histological staining. The levels peaked in the DM 6 month group and were significantly higher compared with the control group. VEGF has been previously reported to be secreted by cancer cells and may downregulate TL1A production by endothelial cells in vitro, and recombinant TL1A has been demonstrated to effectively suppress the angiogenesis and growth of tumors $(17,28)$.
Therefore, VEGF may downregulate the expression of TL1A. The present study demonstrated that the levels of VEGF in the retina and vitreous were higher in DM groups compared with the control group. These data were consistent with previous studies $(24,29)$, which determined that VEGF also stimulated increased leukostasis in the microvessels of the retina and the sticky leukocytes released cytokines or may migrate via the transendothelial route, which may result in a breakdown of the blood-retinal barrier (BRB) and VEGF appeared to be important for promoting intraocular neovascularization in PDR. The present study revealed the ratio of TL1A/VEGF was lower in DM groups compared with the control group. However, the ratio of the DM 6 month group was significantly higher compared with the DM 1 month group. These findings suggested that increased levels of VEGF downregulated the expression levels of TL1A in the early stage of DM (DM 1 month), whilst the organism may overexpress TL1A, as a negative regulator of DM, in order to limit the development and progression of DR (DM 3 month and 6 month groups). Cytokine levels were also investigated in order to determine whether they regulated the expression of TL1A.

Diabetes has been identified to increase the release of retinal inflammatory mediators, such as IL- $1 \beta$, TNF- $\alpha$, VEGF, ICAM-1 and angiotensin II and activate microglial cells in the early stage of DR. TNF- $\alpha$ and IL- $1 \beta$ may induce leukostatis and vascular permeability via activation of the microglia and increased endothelial adhesion $(19,30,31)$. It has been reported that TNF- $\alpha$ is important for the BRB breakdown during DR pathogenesis (32). A previous study determined that anti-angiogenic dose of TNF- $\alpha(30 \mathrm{ng} / \mathrm{ml})$ upregulated the expression of TL1A in endothelial cells (10). Notably, there is clear evidence that the expression of TL1A may be upregulated by TNF- $\alpha$ and IL-1 in HUVECs (18). Therefore, the present study assessed the levels of TNF- $\alpha$ and IL-1 $\beta$ in the different stages of DM. It was determined that the levels of TNF- $\alpha$ and IL- $1 \beta$ were significantly higher in DM 3 month and 6 month groups compared with the control group. Therefore, the levels of TNF- $\alpha$ and IL-1 $\beta$ were higher in DR rats, resulting in increased levels of TL1A.

Previous studies have identified that the elevated serum concentrations of IL- $1 \beta$, TNF- $\alpha$, and VEGF correlated with the presence and severity of DR $(19,21)$. It was suggested that the increase of these cytokines may be an important systemic factor of DR development. However, another study revealed that the vitreous levels of VEGF were not influenced by the VEGF serum concentrations in DR and were not correlated with the presence and severity of DR (33). The results of the current study were consistent with the first study (19), in which levels of VEGF, TNF- $\alpha$, and IL-1 $\beta$ were higher in the DR groups compared with the control group. Changes in TL1A expression levels were partly consistent with those in the retina and vitreous. Briefly, levels of TL1A were significantly lower in the DM 1 and 3 month groups compared with the control group, and significantly increased in the DM 6 month group compared with the DM 3 month group, which was different from the changes of TL1A levels observed in the retina and vitreous. This indicated that the changes of TL1A levels in the serum occurred after those in retina and vitreous. The findings of the present study may provide the rationale required for the development of novel diagnostic strategies of DR. 
In conclusion, the present study determined the changes of TL1A, VEGF, TNF- $\alpha$, and IL-1 $\beta$ expression levels, which occur in the retina, vitreous and serum of rats at different stages of DR. These results suggested that TL1A may be a negative regulator in the development and progression of DR and may be regulated by VEGF, TNF- $\alpha$, and IL- $1 \beta$, and provide additional therapeutic targets for the treatment of DR.

\section{Acknowledgements}

The present study was supported by the National Natural Science Foundation of China (grant nos. 81371038 and 91442124) and the Tianjin Application Infrastructure and Cutting-edge Technology Research Program of China (grant no 10JCZDJC20300) to Dr Hua Yan, and the National Natural Science Foundation of China (grant no. 81500743) to Dr Zhu-Hong Zhang. The views presented in this article do not necessarily reflect those of the U.S. Food and Drug Administration.

\section{References}

1. Ebneter A and Zinkernagel MS: Novelties in diabetic retinopathy. Endocr Dev 31: 84-96, 2016.

2. Tarr JM, Kaul K, Chopra M, Kohner EM and Chibber R: Pathophysiology of diabetic retinopathy. ISRN Ophthalmol 2013: 343560, 2013.

3. Ahsan H: Diabetic retinopathy-biomolecules and multiple pathophysiology. Diabetes Metab Syndr 9: 51-54, 2014.

4. Cheung N, Mitchell P and Wong TY: Diabetic retinopathy. Lancet 376: 124-136, 2010.

5. Tang J and Kern TS: Inflammation in diabetic retinopathy. Prog Retin Eye Res 30: 343-358, 2011.

6. Hsu H and Viney JL: The tale of TL1A in inflammation. Mucosal Immunol 4: 368-370, 2011.

7. Zhang Z and Li LY: TNFSF15 Modulates neovascularization and inflammation. Cancer Microenviron 5: 237-247, 2012.

8. Sethi G, Sung B and Aggarwal BB: Therapeutic potential of VEGI/TL1A in autoimmunity and cancer. Adv Exp Med Biol 647: 207-215, 2009.

9. Chew LJ, Pan H, Yu J, Tian S, Huang WQ, Zhang JY, Pang S and Li LY: A novel secreted splice variant of vascular endothelial cel growth inhibitor. FASEB J 16: 742-744, 2002.

10. Parr C, Gan $\mathrm{CH}$, Watkins $\mathrm{G}$ and Jiang WG: Reduced vascular endothelial growth inhibitor (VEGI) expression is associated with poor prognosis in breast cancer patients. Angiogenesis 9: 73-81, 2006.

11. Yu J, Tian S, Metheny-Barlow L, Chew LJ, Hayes AJ, Pan H, Yu GL and Li LY: Modulation of endothelial cell growth arrest and apoptosis by vascular endothelial growth inhibitor. Circ Res 89: 1161-1167, 2001.

12. Qi JW, Qin TT, Xu LX, Zhang K, Yang GL, Li J, Xiao HY, Zhang ZS and Li LY: TNFSF15 inhibits vasculogenesis by regulating relative levels of membrane-bound and soluble isoforms of VEGF receptor 1. Proc Natl Acad Sci USA 110: 13863-13868, 2013.

13. Stefanini FR, Arevalo JF and Maia M: Bevacizumab for the management of diabetic macular edema. World J Diabetes 4: 19-26, 2013.

14. Gupta N, Mansoor S, Sharma A, Sapkal A, Sheth J, Falatoonzadeh P, Kuppermann B and Kenney M: Diabetic retinopathy and VEGF. Open Ophthalmol J 7: 4-10, 2013.
15. Ho AC, Scott IU, Kim SJ, Brown GC, Brown MM, Ip MS and Recchia FM: Anti-vascular endothelial growth factor pharmacotherapy for diabetic macular edema: A report by the american academy of ophthalmology. Ophthalmology 119: 2179-2188, 2012.

16. Rao RC and Dlouhy BJ: Diabetic retinopathy. N Engl J Med 367: 184,2012

17. Deng W, Gu X, Lu Y, Gu C, Zheng Y, Zhang Z, Chen L, Yao Z and Li LY: Down-modulation of TNFSF15 in ovarian cancer by VEGF and MCP-1 is a pre-requisite for tumor neovascularization. Angiogenesis 15: 71-85, 2012.

18. Kim S and Zhang L: Identification of naturally secreted soluble form of TL1A, a TNF-like cytokine. J Immunol Methods 298: $1-8,2005$

19. Kaul K, Hodgkinson A, Tarr JM, Kohner EM and Chibber R: Is inflammation a common retinal-renal-nerve pathogenic link in diabetes? Curr Diabetes Rev 6: 294-303, 2010.

20. Krady JK, Basu A, Allen CM, Xu Y, LaNoue KF, Gardner TW and Levison SW: Minocycline reduces proinflammatory cytokine expression, microglial activation and caspase-3 activation in a rodent model of diabetic retinopathy. Diabetes 54: 1559-1565, 2005.

21. Koleva-Georgieva DN, Sivkova NP and Terzieva D: Serum inflammatory cytokines IL-1beta, IL-6, TNF-alpha and VEGF have influence on the development of diabetic retinopathy. Folia Med (Plovdiv) 53: 44-50, 2011.

22. National Research Council (US) Committee for the Update of the Guide for the Care and Use of Laboratory Animals: Guide for the Care and Use of Laboratory Animals. 8th edition. National Academies Press, Washington (DC), 2011.

23. Zheng H, Zhang Z, Luo N, Liu Y, Chen Q and Yan H: Increased Th17 cells and IL17 in rats with traumatic optic neuropathy. Mol Med Rep 10: 1954-1958, 2014.

24. Wang J, Chen S, Jiang F, You C, Mao C, Yu J, Han J, Zhang Z and Yan H: Vitreous and plasma VEGF levels as predictive factors in the progression of proliferative diabetic retinopathy after vitrectomy. PLoS One 9: e110531, 2014.

25. Antcliff RJ and Marshall J: The pathogenesis of edema in diabetic maculopathy. Semin Ophthalmol 14: 223-232, 1999.

26. Zhai Y, Ni J, Jiang GW, Lu J, Xing L, Lincoln C, Carter KC, Janat F, Kozak D, Xu S, et al: VEGI, a novel cytokine of the tumor necrosis factor family, is an angiogenesis inhibitor that suppresses the growth of colon carcinomas in vivo. FASEB J 13: 181-189, 1999.

27. Zhai Y, Yu J, Iruela-Arispe L, Huang WQ, Wang Z, Hayes AJ, Lu J, Jiang G, Rojas L, Lippman ME, et al: Inhibition of angiogenesis and breast cancer xenograft tumor growth by VEGI, a novel cytokine of the TNF superfamily. Int J Cancer 82: 131-136, 1999.

28. Hou W, Medynski D, Wu S, Lin X and Li LY: VEGI-192, a new isoform of TNFSF15, specifically eliminates tumor vascular endothelial cells and suppresses tumor growth. Clin Cancer Res 11: 5595-5602, 2005.

29. Penn JS, Madan A, Caldwell RB, Bartoli M, Caldwell RW and Hartnett ME: Vascular endothelial growth factor in eye disease. Prog Retin Eye Res 27: 331-371, 2008.

30. Zeng HY, Green WR and Tso MO: Microglial activation in human diabetic retinopathy. Arch Ophthalmol 126: 227-232, 2008.

31. Wilkinson-Berka JL, Tan G, Jaworski K and Miller AG: Identification of a retinal aldosterone system and the protective effects of mineralocorticoid receptor antagonism on retinal vascular pathology. Circ Res 104: 124-133, 2009.

32. Huang H, Gandhi JK, Zhong X, Wei Y, Gong J, Duh EJ and Vinores SA: TNFalpha is required for late BRB breakdown in diabetic retinopathy and its inhibition prevents leukostasis and protects vessels and neurons from apoptosis. Invest Ophthalmol Vis Sci 52: 1336-1344, 2011.

33. Burgós R, Simo R, Audí L, Mateo C, Mesa J, García-Ramírez M and Carrascosa A: Vitreous levels of vascular endothelial growth factor are not influenced by its serum concentrations in diabetic retinopathy. Diabetologia 40: 1107-1109, 1997. 